\title{
BMJ Open Gonorrhoea and chlamydia diagnosis as an entry point for HIV pre-exposure prophylaxis: a modelling study
}

\author{
Parastu Kasaie, ${ }^{\circledR 1}$ Christina M Schumacher, ${ }^{2}$ Jacky M Jennings, ${ }^{2}$ Stephen A Berry, ${ }^{2}$ \\ Susan A Tuddenham, ${ }^{2}$ Maunank S Shah, ${ }^{2}$ Eli S Rosenberg, ${ }^{3}$ Karen W Hoover, ${ }^{4}$ \\ Thomas L Gift, ${ }^{5}$ Harrell Chesson, ${ }^{5}$ Danielle German, ${ }^{1}$ David W Dowdy ${ }^{1}$
}

To cite: Kasaie $P$,

Schumacher CM, Jennings JM, et al. Gonorrhoea and chlamydia diagnosis as an entry point for HIV preexposure prophylaxis: a modelling study. BMJ Open 2019;9:e023453. doi:10.1136/ bmjopen-2018-023453

- Prepublication history and additional material for this paper are available online. To view these files, please visit the journal online (http://dx.doi org/10.1136/bmjopen-2018023453).

Received 5 June 2018 Revised 14 December 2018 Accepted 20 December 2018

Check for updates

(C) Author(s) (or their employer(s)) 2019. Re-use permitted under CC BY-NC. No commercial re-use. See rights and permissions. Published by BMJ.

For numbered affiliations see end of article.

Correspondence to Dr Parastu Kasaie; pkasaie@jhu.edu

\section{ABSTRACT}

Objectives Neisseria gonorrhoeae (NG) and Chlamydia trachomatis (CT) increase the risk of HIV transmission among men who have sex with men (MSM). Diagnosis of NG/CT may provide an efficient entry point for prevention of HIV through the delivery of pre-exposure prophylaxis (PrEP); however, the additional population-level impact of targeting PrEP to MSM diagnosed with NG/CT is unknown.

Design An agent-based simulation model of NG/CT and HIV cocirculation among MSM calibrated against census data, disease surveillance reports and the US National HIV Behavioral Surveillance study.

Setting Baltimore City, Maryland, USA.

Interventions PrEP implementation was modelled under three alternative scenarios: (1) PrEP delivery at NG/CT diagnosis (targeted delivery), (2) PrEP evaluation at NG/CT screening/testing and (3) PrEP evaluation in the general community (untargeted).

Main outcome The projected incidence of HIV after 20 years of PrEP delivery under two alternatives: when equal numbers of MSM are (1) screened for PrEP or (2) receive PrEP in each year.

Results Assuming $60 \%$ uptake and $60 \%$ adherence, targeting PrEP to MSM diagnosed with NG/CT could reduce HIV incidence among MSM in Baltimore City by $12.4 \%$ (95\% uncertainty range (UR) $10.3 \%$ to $14.4 \%$ ) in 20 years, relative to no PrEP. Expanding the coverage of $\mathrm{NG} / \mathrm{CT}$ screening (such that individuals experience a $50 \%$ annual probability of NG/CT screening and evaluation for PrEP on NG/CT diagnosis) can further increase the impact of targeted PrEP to generate a $22.0 \%$ (95\% UR 20.1\% to $23.9 \%$ ) reduction in HIV incidence within 20 years. When compared with alternative implementation scenarios, PrEP evaluation at NG/CT diagnosis increased impact of PrEP on HIV incidence by $1.5(95 \%$ UR 1.1 to 1.9$)$ times relative to a scenario in which PrEP evaluation happened at the time of NG/CT screening/testing and by 1.6 (95\% UR 1.2 to 2.2) times relative to evaluating random MSM from the community.

Conclusions Targeting MSM infected with NG/CT increases the efficiency and effectiveness of PrEP delivery. If high levels of sexually transmitted infection screening can be achieved at the community level, NG/CT diagnosis may be a highly effective entry point for PrEP initialisation.

\section{Strengths and limitations of this study}

- This study helps to quantify the added value of targeting pre-exposure prophylaxis (PrEP) to men who have sex with men (MSM) diagnosed with Neisseria gonorrhoeae/Chlamydia trachomatis (NG/CT), in terms of population-level impact on disease incidence over time.

- The model is calibrated to a wide array of data including census data, disease surveillance reports and a nationally representative survey of HIV-related behaviours.

- This model offers policy-makers a support tool to estimate and compare the population-level impact of various prevention/control programmes.

- Study findings are limited by simplifying assumptions including (but not limited to) exclusion of other sexually transmitted infections such as syphilis, simplified representation of NG/CT natural history, simplification of sexual networks for NG/CT and HIV transmission, exclusion of HIV transmission through injection drugs or heterosexual sex and exclusion of transgender and bisexual individuals from the simulated population.

- Due to limited data as to whether MSM change their behaviour while taking PrEP, behavioural disinhibition was excluded from this analysis.

\section{BACKGROUND}

Infection with Neisseria gonorrhoeae (NG) and/ or Chlamydia trachomatis (CT) may impact HIV transmission in multiple ways, particularly among men who have sex with men (MSM). From a biological standpoint, NG/ CT infection may increase one's susceptibility to HIV acquisition: rectal infection, in particular, has been linked to an increased risk of HIV acquisition. ${ }^{12}$ HIV and NG/CT also share many risk factors at the individual level (eg, condomless sex) and network level (eg, having sex within a high-prevalence network), such that the three conditions are often epidemiologically linked. ${ }^{3}$ Additionally, HIV-negative men have an increased risk of 
HIV acquisition when in partnership with an HIV-positive partner who is also coinfected with NG/CT. ${ }^{45}$ As a result, better diagnosis and treatment of NG/CT can potentially reduce HIV incidence, ${ }^{6}$ and help to identify individuals at high risk of future HIV infection.

Pre-exposure prophylaxis (PrEP) is part of comprehensive HIV prevention services in which HIV-negative people take daily antiretroviral medication to lower risk of HIV transmission on exposure. The US Centers for Disease Control and Prevention (CDC) has recommended PrEP for HIV-negative individuals at substantial risk of infection. ${ }^{7}$ Among MSM, this includes HIV-negative men who are either diagnosed with a sexually transmitted infection (STI) in the last 6 months, are in an HIV discordant partnership or report a condomless sex act in the last 6 months. Despite this broad recommendation, the potential population-level impact of PrEP remains uncertain. Several barriers exist to the successful implementation of PrEP, including providers' perceived inability to deliver PrEP in primary care settings, ${ }^{8}$ individuals' limited knowledge of PrEP effectiveness, ${ }^{9}$ low self-perceived risk for HIV infection, ${ }^{10}$ patients' difficulty in maintaining adherence $^{11}$ and high costs (at over US $\$ 10000$ per person-year for those without insurance or access to a medication assistance plan). ${ }^{12} 13$

Given these challenges, optimising the efficiency of PrEP delivery is a public health priority. Specifically, it is important to tailor PrEP delivery to those who stand to gain the most from its preventive efficacy. Given the epidemiological link between NG/CT infection and HIV among MSM, new NG/CT diagnoses may serve as a useful means to identify high-risk MSM for PrEP evaluation and delivery. ${ }^{7}$ At present, the impact of such a strategy-in terms of reducing HIV and NG/CT incidence at a population level-is not clear. To address this question, we used surveillance data from Baltimore City (Maryland, USA) to construct an agent-based simulation model of the cotransmission of HIV and NG/CT among MSM, ${ }^{14}$ and applied this model to study the added value of targeting PrEP to MSM diagnosed with NG/CT, in terms of population-level impact on HIV incidence over time.

\section{METHODS}

We base our depiction of HIV on a published agent-based model of HIV transmission among MSM in Baltimore City $^{14}$ (figure 1-top panel), and we extend this model to include coinfection of HIV with NG and CT infections (see section 1 of the online supplementary material).

\section{NG/CT infection}

NG and CT share similarities in natural history, including their acute nature, symptomatology, frequent codiagnosis and cotreatment. ${ }^{15}$ Given these similarities, and for simplicity of modelling, we model NG/CT as a single biological entity. We assume that NG/CT infection may occur at the urethral, rectal or pharyngeal site-each with different probabilities of symptomatic presentation, diagnosis and treatment, and effects on HIV transmission, as shown in table 1 . We include both asymptomatic and symptomatic infection and fit the model to the annual number of diagnoses at each clinical site (urethral, rectal or pharyngeal) among MSM in Baltimore City (see section 2 of the online supplementary material).

\section{STI screening}

In addition to testing of symptomatic NG/CT diagnosis, we also assume screening of asymptomatic individuals as follows (figure 1- bottom panel):

- Guidelines-based screening for HIV and NG/CT: MSM may present to HIV/STI care providers (eg, STI clinics, community health centres, HIV counselling programmes) for a variety of reasons and get tested for HIV and other STIs. We model visits for STI screening as a fixed weekly probability that reflects an individual's age group and sexual activity such that younger MSM with higher propensity of partnerships experience a higher likelihood of visits. ${ }^{16}$ We further assume that NG/CT is always screened at the urethral site, and a proportion of patients are also screened at the rectal and pharyngeal sites (calibrated to match the reported level of NG/CT infections diagnosis at each site among MSM in Baltimore City, as shown in table 1).

- NG/CT screening for HIV-positive MSM in care: Based on CDC recommendations, most MSM who are continuously engaged in HIV care should undergo repeated NG/CT screening at least annually. ${ }^{15}$ More frequent screening, such as screening every 3-6 months, is recommended for high-risk MSM, including those with an NG/CT diagnosis in the last year. Based on data from Baltimore City and a conservative estimate, we assume $40 \%$ adherence to these guidelines. ${ }^{18} 19$

\section{HIV testing}

In addition to combined HIV/STI testing that takes place as part of STI screening, we assume that all MSM experience an additional probability of HIV testing (in excess of testing through the STI programme) and calibrate this probability to match the reported level of HIV diagnosis among MSM in Baltimore City.

\section{Calibration}

The model was calibrated against aggregate estimates of HIV and NG/CT incidence and prevalence, as well as the estimated continuum of HIV care, in Baltimore City. Calibration targets pertaining to NG/CT epidemiology are derived from data on gonorrhoea surveillance and STI clinic visits collected by the Baltimore City Health Department as part of the STD Surveillance Network Project (see section 2 of the online supplementary material).

\section{Pre-exposure prophylaxis}

Our primary outcome for this analysis is the projected incidence of HIV after 20 years of delivering PrEP to MSM. We measure this outcome in three different PrEP delivery scenarios, selected for purposes of evaluating the 


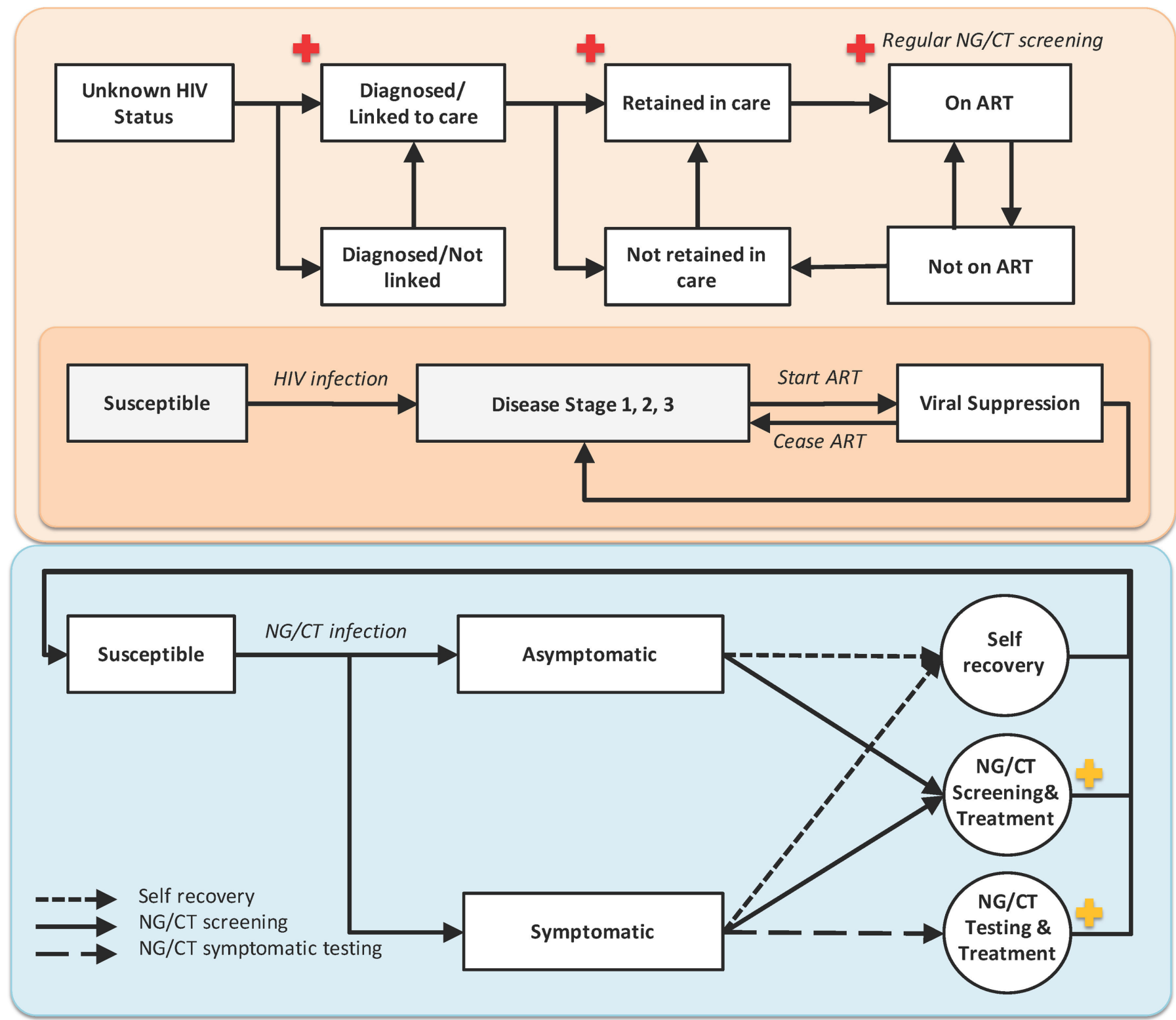

Figure 1 An agent-based model of Neisseria gonorrhoea/Chlamydia trachomatis (NG/CT) and HIV cotransmission. The top panel represents the HIV care continuum and natural history: On infection with HIV, individuals serially progress through three disease stages over time; this progression can be halted by initiation of antiretroviral therapy (ART), which is assumed to result - if taken -in viral suppression within 4-24 weeks (see table 1). ${ }^{29}$ We assume, for simplicity, that engagement in care involves initiation of ART (as episodes of care engagement not resulting in ART initiation do not affect HIV transmission in the model). HIV-positive individuals in care are assumed to undergo regular screening for NG/CT (marked in red) subject to patients presenting for scheduled visits and clinician decision to screen. The bottom panel represents the natural history of NG/CT: infection may be symptomatic or asymptomatic, individuals remain infectious until diagnosis and treatment (which can occur either through symptomatic presentation to care or routine screening of asymptomatic individuals) or spontaneous resolution. On diagnosis with incident NG/CT, we assume that individuals are also screened for HIV infection (marked in yellow); if HIVnegative, we consider the possibility of PrEP delivery in this analysis. PrEP, pre-exposure prophylaxis.

added benefit of targeting PrEP at individuals diagnosed with NG/CT. In all three scenarios, indication for PrEP use (eligibility) is considered in accordance with CDC recommendations and Baltimore City PrEP guidelines ${ }^{20}$ (see section 1 of the online supplementary material) and includes HIV-negative individuals who are diagnosed with $\mathrm{NG} / \mathrm{CT}$ in the last 6 months, live in a serodiscordant partnership or report an unprotected sex act or a new casual partnership in the last 6 months. The three scenarios are thus:

- PrEP delivery at NG/CT diagnosis ('targeted' strategy and primary analysis): all MSM diagnosed with NG/CT are offered PrEP at the time of diagnosis.

- PrEP evaluation at NG/CT screening/testing ('at-testing'): PrEP eligibility is evaluated at the time 
Table 1 List of selected simulation parameters and calibration targets

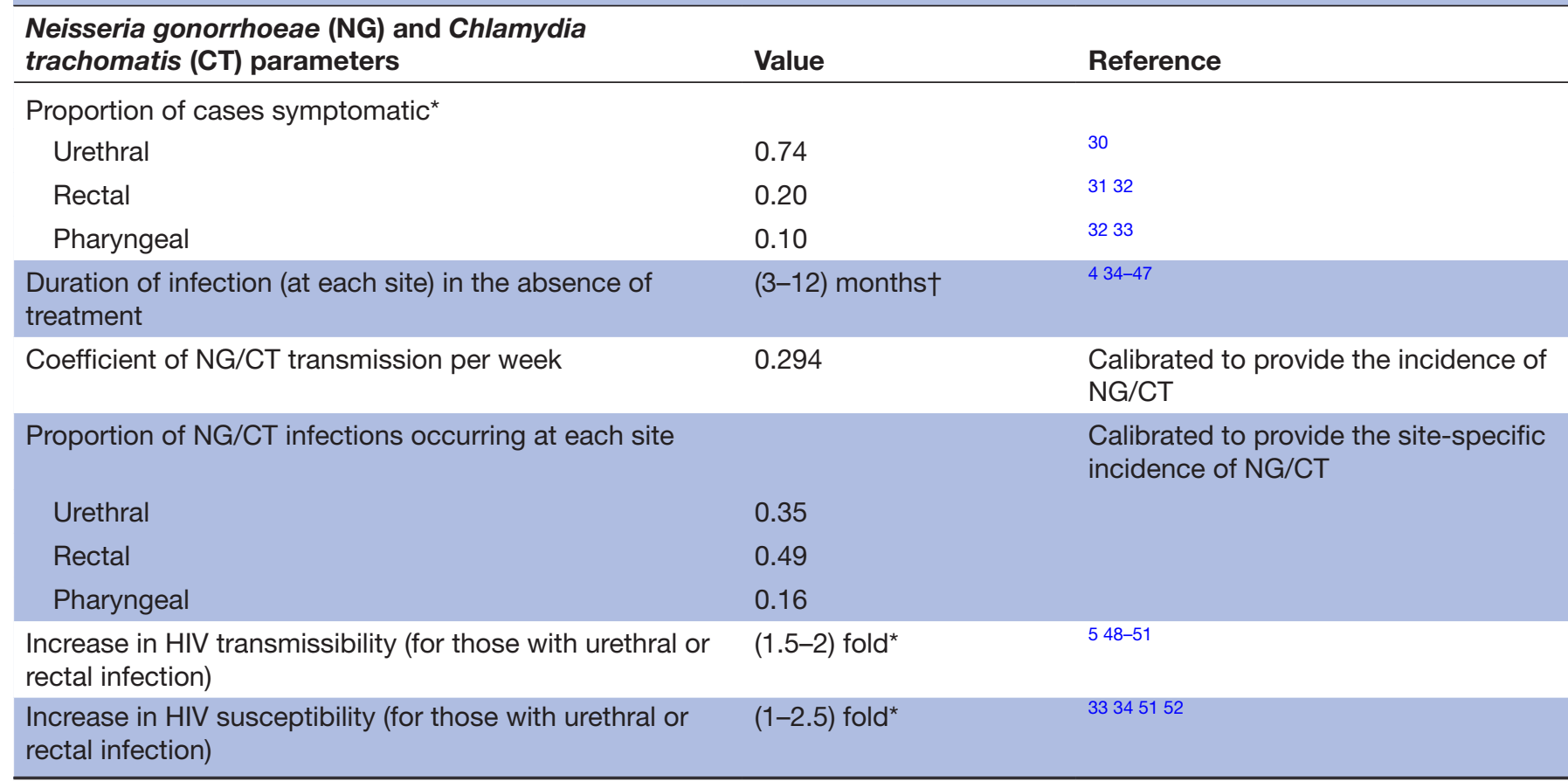

\begin{tabular}{|c|c|c|}
\hline NG/CT calibration targets & Mean¥ (Range) & \\
\hline $\begin{array}{l}\text { Number of annual NG/CT diagnosis among MSM in } \\
\text { Baltimore City }\end{array}$ & & \multirow{7}{*}{$\begin{array}{l}\text { Values estimated from local data on } \\
\text { gonorrhoea surveillance and STI clinic } \\
\text { visits in Baltimore City (see section } 2 \text { in } \\
\text { the online supplementary material) }\end{array}$} \\
\hline Rectal & 25 (18-33) & \\
\hline Pharyngeal & $42(23-60)$ & \\
\hline \multicolumn{2}{|l|}{$\begin{array}{l}\text { Site-specific annual number of incident NG/CT cases } \\
\text { among MSM in Baltimore City }\end{array}$} & \\
\hline Urethral & 944 (753 - 1135) & \\
\hline Rectal & $1251(998-1505)$ & \\
\hline Pharyngeal & 409 (326 - 492) & \\
\hline
\end{tabular}

\section{HIV parameters}

Disease duration

$\begin{array}{lll}\text { Acute } & (6-9) \text { weeks }^{*} & 5354 \\ \text { Chronic } & (8-10) \text { years }^{*} & 5556 \\ \text { Late stage§ } & (1-3) \text { years* } & 55-57\end{array}$

Mortality ratef

Acute and chronic, no ART 5 per 1000 person-years $\quad$ 58-60

Late stage, no ART 1/duration of late stage

Reduction in mortality due to ART 0.58

Time from ART discontinuation to pre-ART CD4 nadirf $\quad$ ART treatment duration 61-64

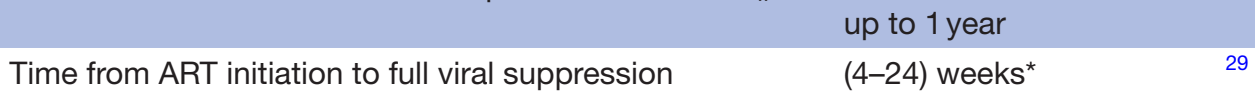

$\begin{array}{ll}\text { Average viral load (log10 copies } / \mathrm{mL}) & 55 \\ \text { Acute, no ART } & 6.5 \\ \text { Chronic, no ART } & 4.5 \\ \text { Late stage, no ART } & 5 \\ \text { On ART, partially suppressed } & 3.5 \\ \text { On ART, fully suppressed } & 1.5\end{array}$


Table 1 Continued

\begin{tabular}{lll}
\hline HIV parameters & & \\
\hline Infectiousness per sexual contact & $2.45^{(\log (\mathrm{VL})-4.5)}$ & 55 \\
Weekly probability of engagement in HIV care & 0.006 & $65-67$ \\
Weekly probability of ART discontinuation & 0.015 & 69 \\
Gap in care after ART discontinuation & 26 weeks & 70 \\
Relative probability of accessing HIV care among black & 0.5 & 71 \\
MSM compared with white MSM & & 71 \\
\hline HIV calibration targets & & \\
\hline HIV prevalence & 0.22 per 100000 person- & 71 \\
HIV continuum of care: proportion of cases & year & \\
Diagnosed & & \\
Linked to care & 0.86 & \\
Engaged in care & 0.62 & \\
On ART & 0.5 & 0.39 \\
Virally suppressed & 0.27 & \\
\hline
\end{tabular}

*Values represent a pooled estimate of the reported measures for NG and CT infections.

†Values are selected over uniform distributions across the ranges presented.

¥Values represent the reported levels of NG/CT diagnosis among Baltimore City's MSM, and they are likely to underestimate the proportion of ongoing rectal and pharyngeal infections. We, therefore, consider such potential underestimation in estimating the annual incidence of NG/CT (see section 2 of the online supplementary material) and have calibrated the model to represent realistic levels of prevalence (see the section on population overview in the main text).

$\S$ Mortality rate in late stage is defined as $1 /$ (duration of late-stage disease).

IInfectiousness assumed equal to that of the chronic disease.

ART, antiretroviral therapy; MSM, men who have sex with men; STI, sexually transmitted infection.

of screening/testing for NG/CT, and all eligible individuals are offered PrEP.

- Untargeted PrEP: PrEP eligibility is evaluated at random, and all eligible MSM are offered PrEP.

All else being equal, increasing the number of MSM on PrEP will result in larger effects on HIV incidence (as more people are protected from HIV transmission). However, for a given number of MSM screened-or a given number of MSM on PrEP (eg, if resource constraints are such that not all MSM meeting the criteria for PrEP can be placed/maintained on PrEP) — targeting PrEP to those screened for/diagnosed with NG/CT may be more efficient. Our primary aim was to quantify the extent of this gain in efficiency; thus, we compared scenarios in which the same number of MSM would be evaluated for PrEP, or alternatively the same number of MSM would be maintained on PrEP. Furthermore, to illustrate the potential impact of reaching highly ambitious targets for improved STI screening, we considered a hypothetical scenario for improving the underlying level of NG/CT screening (such that individual MSM not on PrEP experience a $50 \%$ annual probability of NG/CT screening and evaluation for PrEP on NG/CT diagnosis), and studied the additional gain in effectiveness of NG/CT-targeted PrEP under this assumption.

In all scenarios, we assume that PrEP eligibility is reassessed every 3 months among patients receiving PrEP, and those who remain eligible for PrEP continue to receive it over time. Furthermore, we assume that in each scenario, a given proportion of eligible MSM who are offered PrEP will initiate prophylaxis (PrEP uptake ranging $(0 \%-100 \%)$ ) and adhere to it (PrEP adherence ranging $(0 \%-100 \%)$ ), with adherence defined as taking a sufficient number of doses to protect against HIV transmission in the specified percentatge of potential transmission events. ${ }^{21}$ As a criterion for initiation of PrEP, all eligible MSM are also screened and treated for NG/CT infection before starting PrEP.

\section{Sensitivity analysis}

A variety of sensitivity analyses were performed with the model. Using the HIV incidence at 10 years in the absence and presence of PrEP (via all three scenarios) as the main output of interest, one-way sensitivity analyses were performed to the variation of all model parameters to $\pm 25 \%$ of their original value. We also varied condom usage among MSM on PrEP to model behavioural disinhibition (section 3 of the online supplementary material).

\section{Patient and public involvement}

Patients and/or public were not involved in this study.

\section{RESULTS}

\section{Population overview}

The simulation models a population of 15000 MSM in Baltimore City, projecting an average of $215(95 \%$ 


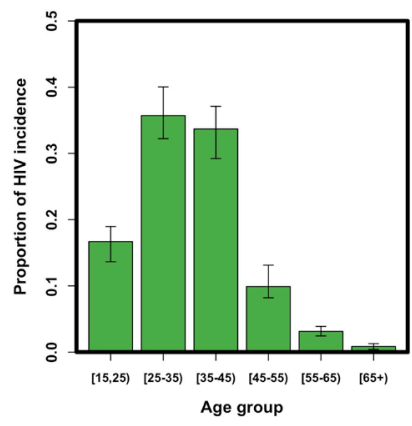

(c)

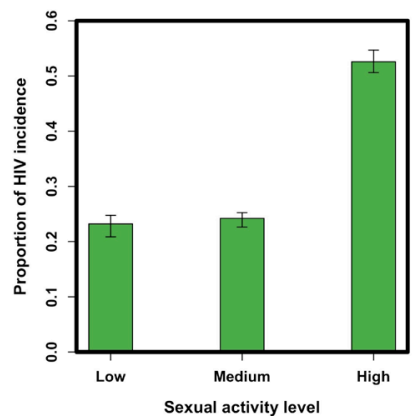

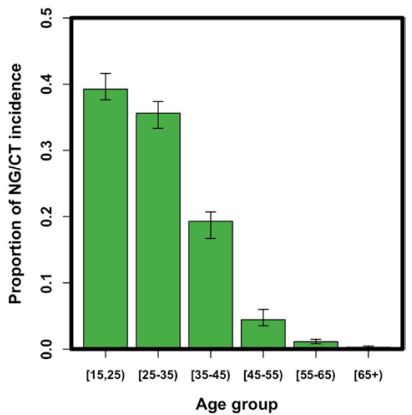

(D)

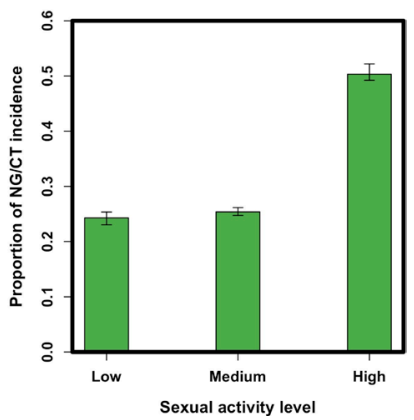

Figure 2 Model projections of the distribution of new infections by age and sexual activity level. Shown on the $y$-axes are the distribution of HIV (A) and NG/CT (B) incidence by age group and the distribution of HIV (C) and NG/CT (D) incidence by the sexual activity level. Bars represent the mean values of simulations (in green) with error bars representing the $95 \%$ uncertainty range of observations around each simulated measure. NG/CT, Neisseria gonorrhoeae/Chlamydia trachomatis.

uncertainty range (UR): 181 to 251) incident HIV cases per year. Within this population, the coepidemic of NG/ CT was calibrated to 2598 (2204-2996) incident cases annually among which $35.0 \%$ (33.4\% to $36.5 \%)$ of cases appear with urethral infection, $49.0 \%(47.4 \%$ to $50.6 \%)$ with non-urethral/rectal infection and $16.0 \%(14.8 \%$ to $17.2 \%$ ) with pharyngeal-only infection. Point prevalence of NG/CT infection was estimated as $9.9 \%(8.4 \%$ to $11.5 \%$ ), with $68.0 \%$ (63.5\% to $72.5 \%)$ of infections occurring among black MSM (accounting for $58 \%$ of the MSM population). New infections occurred primarily in younger individuals, with $74 \%(72 \%$ to $78 \%)$ of new $\mathrm{NG} / \mathrm{CT}$ infections occurring in MSM younger than 35 years old, and $69 \%$ (66\% to $72 \%$ ) of new HIV infections occurring among MSM between the ages of 25 and 45 (figure 2A,B). Over half of new HIV and NG/CT infections occurred among MSM in the high sexual activity class, which accounted for $33 \%$ of the simulated population (figure $2 \mathrm{C}, \mathrm{D})$. Overall, $81.5 \%(81.0 \%$ to $82.1 \%$ ) of MSM diagnosed with NG/CT in the model were tested on the basis of symptomatic presentation (rather than asymptomatic screening), and 20\% (18.0\% to $22.0 \%)$ of incident NG/CT cases were coinfected with HIV.

\section{Epidemiological impact of PrEP at NG/CT diagnosis}

At baseline and in the absence of PrEP (steady-state equilibrium), 361 (95\% UR 298 to 427) MSM were annually

(A)

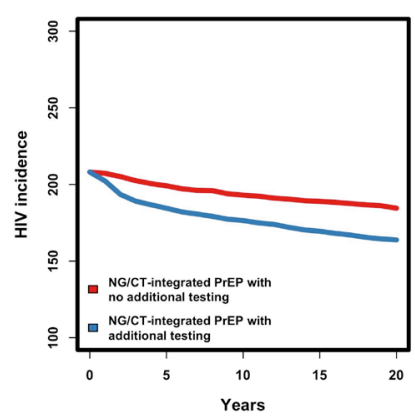

c

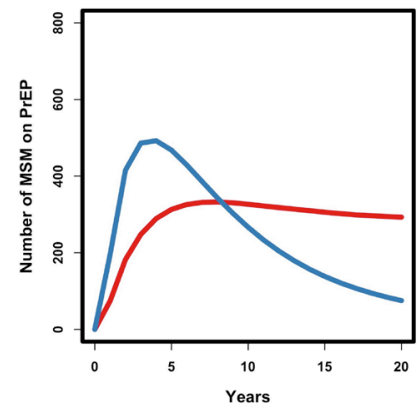

(B)

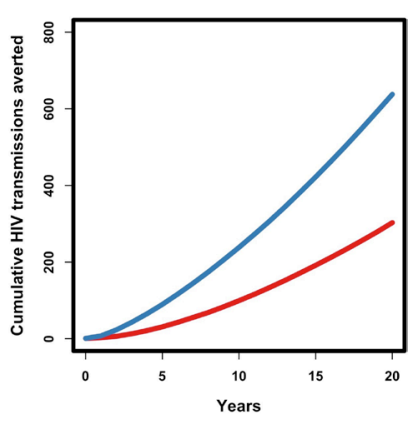

(D)

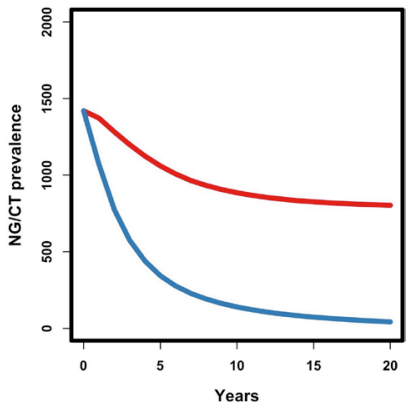

Figure 3 Impact of NG/CT-integrated PrEP, according to frequency of NG/CT screening/testing. Shown on the $y$-axes are the annual incidence of HIV (A), cumulative number of HIV transmissions averted (B), number of MSM on $\operatorname{PrEP}(\mathrm{C})$ and NG/CT prevalence (D). The red line depicts a scenario in which all MSM currently diagnosed with NG/ CT are placed on PrEP with $60 \%$ uptake and adherence (NG/ CT-integrated PrEP scenario in the main text), and the blue line shows a hypothetical scenario in which $50 \%$ of MSM are additionally screened for NG/CT every year, with those testing positive for NG/CT also offered PrEP. MSM, men who have sex with men; NG/CT, Neisseria gonorrhoeae/Chlamydia trachomatis; PrEP, pre-exposure prophylaxis.

diagnosed and treated for NG/CT infection (calibrated). If $60 \%$ of MSM diagnosed with NG/CT could be started on PrEP (ie, uptake $=60 \%$ ) and maintained at a degree to which $60 \%$ of subsequent HIV infections were averted (ie, adherence $=60 \%$ ), HIV incidence was estimated to decline by $12.4 \%$ ( $10.3 \%$ to $14.4 \%$ ) over 20 years (figure $3 \mathrm{~A}$ ). This corresponds to averting 318 (253-385) potential HIV transmissions through 5808 (5730-5886) personyears of PrEP delivered or 5479 (4330-6632) infections averted per 100000 person-years of PrEP (figure 3B). Under the current level of $\mathrm{NG} / \mathrm{CT}$ diagnosis, the number of MSM receiving PrEP is projected to increase through the first 8 years of the programme (reaching a total of 332 (327-338) MSM on PrEP) and to fall afterwards with a declining incidence of NG/CT (figure 3C). Due to the increased level of NG/CT screening/treatment among those on PrEP (through reassessment every 3 months), the prevalence of $\mathrm{NG} / \mathrm{CT}$ was estimated to decline by $43.3 \%$ ( $41.6 \%$ to $44.9 \%$ ) over 20 years of PrEP implementation (figure 3D).

The impact of PrEP on HIV incidence can be further increased by expanding the coverage of NG/CT screening at the community level. In our baseline model, 
(A)

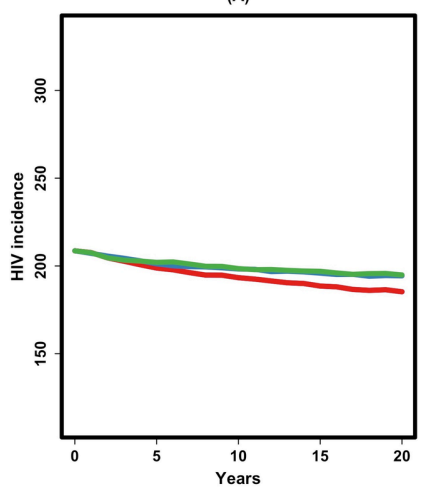

(E)

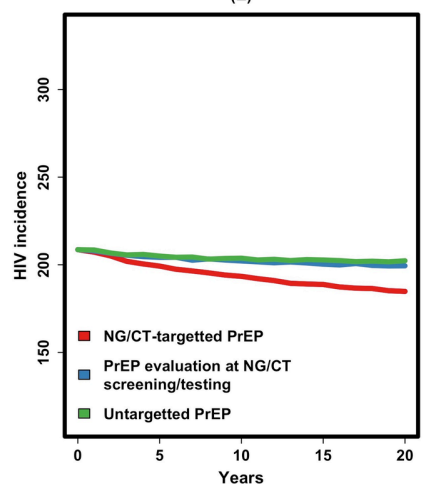

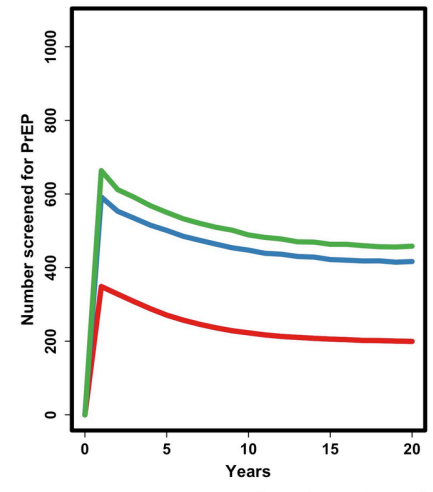

(F)

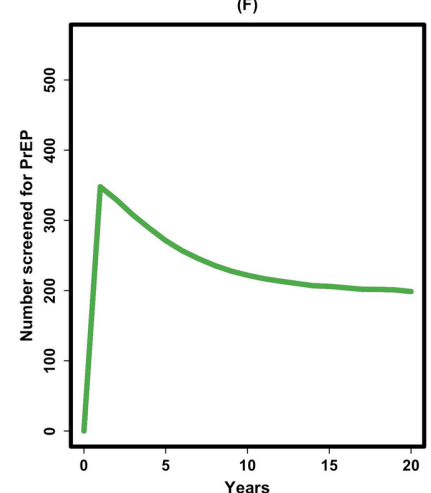

(C)

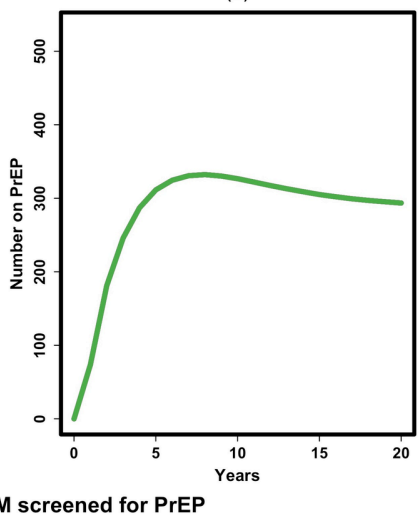

(G)

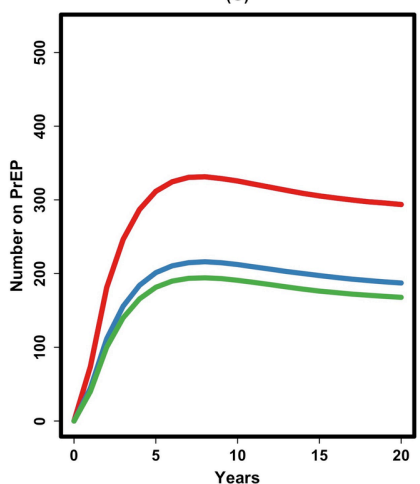

(D)

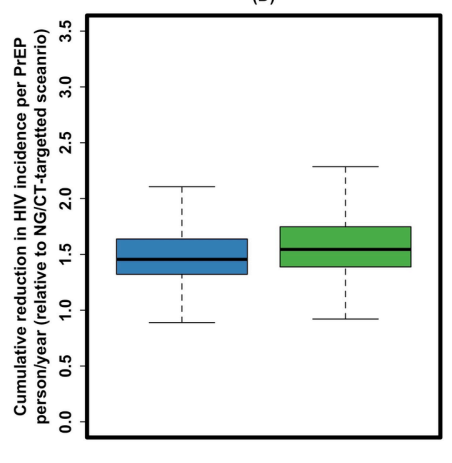

(H)

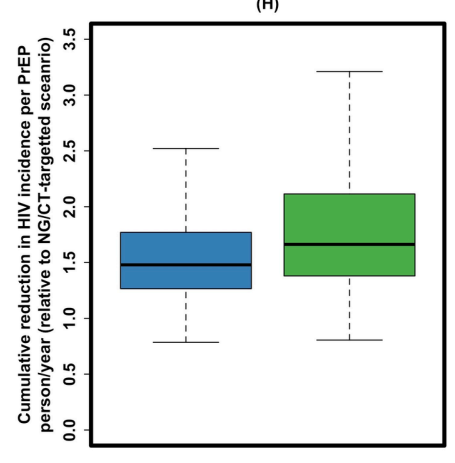

Figure 4 Relative impact of NG/CT-integrated PrEP. Shown in this figure is the relative impact of NG/CT-integrated PrEP (in red, also corresponding to the red line in figure 3), compared against PrEP evaluation at NG/CT screening/testing (in blue) and untargeted PrEP (in green), with full description of these scenarios given in the manuscript text. In the first set of experiments, the three strategies are compared under the assumption that the same number of MSM would receive PrEP (panel A to D), or the same number of MSM would be screened for PrEP (panel E to H). Panel A gives the annual incidence of HIV, panel B the number of MSM approached for PrEP, panel $\mathrm{C}$ the number of MSM on PrEP at any point in time (all three lines overlapping) and panel $D$ the cumulative reduction in HIV incidence per PrEP person-year in untargeted scenarios relative to NG/CT-targeted scenario (similar pattern in panel E through H). MSM, men who have sex with men; NG/CT, Neisseria gonorrhoeae/Chlamydia trachomatis; PrEP, pre-exposure prophylaxis.

$25.0 \%(95 \%$ UR $24.0 \%$ to $26.0 \%)$ of MSM undergo $\mathrm{NG} / \mathrm{CT}$ screening/testing at least once annually (CDC recommendation). In an expanded-screening scenario in which all MSM experienced a $50 \%$ probability of screening for NG/CT annually, we projected a $180 \%$ increase in the baseline estimate of 4033 (3883-4182) annual NG/CT testing/screening events. Offering PrEP to those testing positive for NG/CT subsequently generated a $22.0 \%(20.1 \%$ to $23.9 \%)$ decline in HIV incidence over 20 years, corresponding to 648 (589-710) potential HIV transmissions averted. For further information on levels of uncertainty in these results, see section 4 of the online supplementary material.

\section{Relative impact of targeted versus untargeted PrEP}

NG/CT-integrated PrEP increased the efficiency of PrEP delivery in at least two ways (figure 4A). First, a higher percentage of MSM were eligible for PrEP among those evaluated for PrEP (figure 4B, C). In our model, $71.1 \%$ (95\% UR $65.0 \%$ to $77.2 \%)$ of all MSM diagnosed with NG/CT were eligible to receive PrEP (as $29 \%$ of this population is HIV-positive), compared with $45.2 \%$ (43.2\% to
$48.2 \%)$ of MSM screened for NG/CT and $41.3 \%(39.1 \%$ to $43.5 \%$ ) of randomly selected MSM. Second, providing PrEP to MSM diagnosed with NG/CT targets individuals at higher risk of potential HIV infection (due to both biological factors and high-risk behaviour), such thatunder the baseline assumption of equal numbers of people receiving PrEP - the impact of NG/CT-targeted PrEP on HIV incidence was greater than the other two scenarios (figure 4D). Specifically, over 20 years of implementation, targeting PrEP to MSM diagnosed with NG/CT infection increased the impact of PrEP by 1.5 (1.1-1.9) times relative to PrEP evaluation at NG/CT screening/testing, and by 1.6 (1.2-2.2) times relative to untargeted PrEP. In another comparison, if the same number of individuals were evaluated for PrEP, the efficacy of NG/CT-integrated PrEP was increased even further relative to other scenarios (figure $4 \mathrm{E}$ through $4 \mathrm{H}$ ).

In one-way sensitivity analyses, the projected HIV incidence at 10 years in the absence of $\mathrm{PrEP}$ was sensitive to parameters relating to $\mathrm{HIV}$ and $\mathrm{NG} / \mathrm{CT}$ transmission (including level of HIV viral load, condom use and 
condom effectiveness) and parameters describing overall sexual activity (including the probabilities of starting new partnerships and the level of sexual activity in the most sexually active class). A similar variation in HIV incidence was observed in scenarios modelling PrEP evaluation at the time of NG/CT diagnosis, NG/CT screening or at random. Impact of PrEP in terms of reduction in HIV incidence in all scenarios relative to baseline was robust to reasonable variation of most model parameters (section 3 of the online supplementary material).

\section{DISCUSSION}

This agent-based simulation of HIV transmission among MSM suggests that screening for NG/CT may be an important and efficient entry point for PrEP evaluation and delivery. Specifically, if all MSM who currently test positive for NG/CT could be offered PrEP-assuming $60 \%$ uptake and sufficient adherence to maintain $60 \%$ protection-HIV incidence could be reduced by approximately $12 \%$, averting one HIV infection annually per 1000 MSM population, with fewer than 20 per 1000 taking PrEP every year. On the basis of infections averted per PrEP dose delivered, providing PrEP to MSM with NG/CT diagnosis is nearly twice as efficient as providing PrEP randomly among eligible MSM. Thus, the use of $\mathrm{NG} / \mathrm{CT}$ diagnosis as an entry point is a highly efficient and feasible mechanism for PrEP delivery. If NG/CT screening could be expanded to $50 \%$ of MSM every year (with PrEP offered only to those testing positive), this impact could be more than doubled. Given this substantial potential impact, it will be important to assess willingness and uptake and identify best practices to support PrEP uptake and adherence among MSM diagnosed with $\mathrm{NG} / \mathrm{CT}$.

These findings are consistent with other studies of PrEP delivery among MSM. ${ }^{22}{ }^{23}$ Previous studies have shown that the population-level impact of PrEP depends strongly on PrEP uptake and adherence, ${ }^{14}{ }^{22}$ as suggested in our study as well. Importantly, NG/CT diagnosis may be useful in this regard, as MSM who have recently been diagnosed with an STI may be more aware of their HIV risk and more likely to accept and initiate PrEP. Past research has shown that HIV interventions may be more effective when they are conducted or initiated at the time of an STI diagnosis. ${ }^{24}$ Initiation of PrEP simultaneously with $\mathrm{NG} / \mathrm{CT}$ diagnosis may also be a clinically feasible approach-as an STI diagnosis is already likely to prompt an HIV test (if not already performed), and MSM who are diagnosed with NG/CT have at least some level of healthcare access. Unlike performing detailed sexual histories, offering PrEP to all HIV-negative MSM diagnosed with $\mathrm{NG} / \mathrm{CT}$ is a simple guideline that is easy for most clinicians to follow. ${ }^{25}{ }^{26}$ Further research is needed to assess the feasibility of this approach in the field, especially in ascertaining the degree to which the continuum of PrEP care (including linkage to care and longer term maintenance on PrEP) can be maintained in this population.
Furthermore, the potential trade-off between the positive impact of PrEP on STI prevalence through enhanced screening and its negative impact through behavioural disinhibition (if MSM on PrEP adopt riskier sexual behaviours) merits further investigation. Additional implementation research is also needed to identify effective mechanisms for improving adherence to CDC PrEP guidelines and overcoming barriers to acceptance and uptake of PrEP such as lack of awareness, lack of access, financial strain and stigma. ${ }^{11} 27$

As with any modelling analysis, our findings are limited by necessary simplifying assumptions. Given the overlap in clinical practice for treating NG and CT and the substantial uncertainty regarding the natural history of the two infections (eg, duration of infectiousness, propensity toward asymptomatic infection), we have combined these infections as a single entity (NG/CT) and have used composite parameter values to describe the natural history of both diseases. However, there are still important differences between NG and CT, and to the extent that the natural history of each disease may differ, our findings may overestimate or underestimate the impact of PrEP targeted at these STIs. For example, an infection with a shorter infectious period and a higher transmission probability per sex act will concentrate more strongly on high-risk networks and may provide a more effective entry point for HIV PrEP. Further research can extend our analysis by considering the impact of each disease separately on HIV transmission dynamics. Furthermore, due to limited data on site-specific transmission dynamics (eg, relative frequency of oral-only vs oral-plus-anal vs anal-only sex among MSM in Baltimore), we adopted a simplified approach that does not fully capture the complete transmission dynamics but should result in the appropriate distributions of $\mathrm{NG} / \mathrm{CT}$ infections at each anatomical site. Additional simplifying assumptions used in the underlying HIV simulation model include applying the same sexual network for NG/CT and HIV transmission; simplification of sexual networks as comprising only stable and casual partnerships; simplified definition of sexual activity classes as a lifetime attribute among MSM; exclusion of serosorting by HIV status, sexual activity class or PrEP; exclusion of HIV transmission through injection drugs or heterosexual sex and exclusion of transgender individuals from the simulated population. To the extent that these dynamics result in a higher concentration of NG/CT among MSM at high risk for HIV infection, our model may underestimate the impact of STI-targeted PrEP.

We excluded the potential existence of behavioural disinhibition for MSM on PrEP in the main analysis and applied a simplified approach for modelling the combined role of PrEP uptake/adherence for HIV protection. In additional sensitivity analyses, we studied the impact of decreased condom use among MSM on PrEP on the outcome of NG/CT-targeted PrEP, and the relative efficacy of STI-targeted scenario compared with the other comparators (see section 3 of the online supplementary 
material). As expected, the projected impact of $\mathrm{NG} /$ CT-targeted PrEP on the incidence of HIV and NG/CT declined with reduced levels of condom use among PrEP users. This further highlights the need for additional behavioural surveillance data characterising changes in the level of condom use and risky behaviours among PrEP users in local settings. Despite this behaviour, the main outcome of our analysis (increased effectiveness of PrEP implementation through an NG/CT-targeted approach) remained robust to variation in the rate of condom use reduction.

There are strong racial disparities in HIV incidence and healthcare access in the USA, such that the highest risk populations may be the ones least likely to have access to $\mathrm{PrEP}^{28}$; these disparities were not included in our simplified cascade of PrEP. Our model calibration was limited to the scope of local surveillance data, and available literature for values lacking direct empiric estimates from Baltimore City (eg, probability of symptomatic infection). We also assumed a future trajectory of HIV infection in the future that represents continuation of current trends; this trajectory is unlikely to remain constant for the next 20 years but may help to provide a useful conceptual construct for present-day decision-making, which is the ultimate goal of this analysis. Our results are further limited by exclusion of syphilis infection, another STI that is often transmitted in the same populations and may affect transmission and acquisition of HIV. Finally, we did not incorporate cost or other resource constraints into the present model; future analyses could evaluate the efficiency of NG/CT-targeted PrEP delivery from a cost-effectiveness or budget impact perspective.

In summary, this stochastic agent-based model representing the codynamics of NG/CT and HIV transmission among MSM suggests that NG/CT diagnosis may serve as an efficient and effective entry point for PrEP. If linkage between STI and HIV control programmes can be effectively developed, further investment in NG/CT screening (followed by PrEP initiation) can have major impact, on the incidence and prevalence of NG/CT, and on transmission of HIV - potentially averting up to $20 \%$ of all HIV infections through NG/CT-targeted PrEP alone. Future analyses could evaluate whether such approaches could even be cost saving in the long term. Ultimately, ending the HIV epidemic in MSM populations will require a combination of multiple activities, including strengthening the continuum of HIV care, ensuring continued access to clinical services and prevention through both behavioural approaches (eg, condom use) and PrEP. Using NG/CT diagnosis as an entry point for PrEP initiation may serve as an important component of such a combined prevention approach.

\section{Author affiliations}

${ }^{1}$ Bloomberg School of Public Health, Johns Hopkins University, Baltimore, Maryland, USA

${ }^{2}$ School of Medicine, Johns Hopkins University, Baltimore, Maryland, USA

${ }^{3}$ Rollins School of Public Health, Emory University, Atlanta, Georgia, USA
${ }^{4}$ Division of HIV/AIDS Prevention, National Center for HIV/AIDS, Viral Hepatitis, STD, and TB Prevention, Centers for Disease Control and Prevention, Atlanta, Georgia, USA

${ }^{5}$ Division of STD Prevention, National Center for HIV/AIDS, Viral Hepatitis, STD, and TB Prevention, Atlanta, Georgia, USA

Acknowledgements We thank members of the scientific and public health advisory groups of the Coalition for Applied Modeling for Prevention project for their input on this study, and specifically those members who reviewed a previous version of this manuscript: Gregory Felzien and Jane Kelly. We acknowledge funding from National Center for HIV, Viral Hepatitis, STDs and TB Prevention Epidemic and Economic Modeling Agreement (NEEMA) (U38 PS004646-01).

Contributors Designed the study (PK and DWD); wrote the model code (PK); provided data (CMS, JMJ, SAT and DG); analysed the data (PK); supervised the analyses (DWD); reviewed results (MSS, SAB, KWH, TLG and HC); wrote the first draft of the manuscript (PK); revised the manuscript and contributed intellectual content (PK, SAB, MSS, ESR, KWH, TLG and HC); all authors saw and approved the final manuscript.

Funding This work was supported by: US Centers for Disease Control and Prevention (Enhancing Models of HIV, Viral Hepatitis, STIs and Tuberculosis to Inform and Improve Public Health Impact)—PK, DWD and MSS. US Centers for Disease Control and Prevention (STD Surveillance Network PS13-1306)—CMS. Johns Hopkins University Center for AIDS Research (P30Al094189)—PK.

Competing interests None declared.

Patient consent for publication Not required.

Provenance and peer review Not commissioned; externally peer reviewed.

Data sharing statement Additional data are presented in the online supplementary material.

Open access This is an open access article distributed in accordance with the Creative Commons Attribution Non Commercial (CC BY-NC 4.0) license, which permits others to distribute, remix, adapt, build upon this work non-commercially, and license their derivative works on different terms, provided the original work is properly cited, appropriate credit is given, any changes made indicated, and the use is non-commercial. See: http://creativecommons.org/licenses/by-nc/4.0/.

\section{REFERENCES}

1. Katz DA, Dombrowski JC, Bell TR, et al. HIV Incidence among men who have sex with men after diagnosis with sexually transmitted infections. Sex Transm Dis 2016;43:249-54.

2. Pathela P, Braunstein SL, Blank S, et al. HIV incidence among men with and those without sexually transmitted rectal infections: estimates from matching against an HIV case registry. Clin Infect Dis 2013;57:1203-9.

3. Ward H, Rönn M. Contribution of sexually transmitted infections to the sexual transmission of HIV. Curr Opin HIV AIDS 2010;5:305-10.

4. Chesson HW, Pinkerton SD. Sexually transmitted diseases and the increased risk for HIV transmission: implications for costeffectiveness analyses of sexually transmitted disease prevention interventions. J Acquir Immune Defic Syndr 2000;24:48-56.

5. Fleming DT, Wasserheit JN. From epidemiological synergy to public health policy and practice: the contribution of other sexually transmitted diseases to sexual transmission of HIV infection. Sex Transm Infect 1999;75:3-17.

6. Grosskurth H, Mosha F, Todd J, et al. Impact of improved treatment of sexually transmitted diseases on HIV infection in rural Tanzania: randomised controlled trial. Lancet 1995;346:530-6.

7. Centers for Disease Control Prevention. Preexposure Prophylaxis for the Prevention of HIV Infection in the United States - 2014 Clinical practice guideline. 2014

8. Krakower D, Ware N, Mitty JA, et al. HIV providers' perceived barriers and facilitators to implementing pre-exposure prophylaxis in care settings: a qualitative study. AIDS Behav 2014;18:1712-21.

9. Eaton LA, Driffin DD, Bauermeister J, et al. Minimal awareness and stalled uptake of pre-exposure prophylaxis (prep) among at risk, HIV-Negative, black men who have sex with men. AIDS Patient Care STDS 2015;29:423-9.

10. Galindo GR, Walker JJ, Hazelton P, et al. Community member perspectives from transgender women and men who have sex with men on pre-exposure prophylaxis as an HIV prevention strategy: implications for implementation. Implement Sci 2012;7:116. 
11. Golub SA, Gamarel KE, Rendina HJ, et al. From efficacy to effectiveness: facilitators and barriers to PrEP acceptability and motivations for adherence among MSM and transgender women in New York City. AIDS Patient Care STDS 2013:27:248-54.

12. Juusola JL, Brandeau ML, Owens DK, et al. The cost-effectiveness of preexposure prophylaxis for HIV prevention in the United States in men who have sex with men. Ann Intern Med 2012;156:541-50.

13. Koppenhaver RT, Sorensen SW, Farnham PG, et al. The costeffectiveness of pre-exposure prophylaxis in men who have sex with men in the United States: an epidemic model. J Acquir Immune Defic Syndr 2011;58:e51-2.

14. Kasaie P, Pennington J, Berry SA, et al. The impact of pre-exposure prophylaxis among men who have sex Withmen in Baltimore City: an agent-based simulation. JAIDS J Acquir Immune Defic Syndr 2017:75:175-83.

15. Workowski KA, Bolan GA. Centers for Disease Control and Prevention. Sexually transmitted diseases treatment guidelines, 2015. MMWR Recomm Rep 2015;64(RR-03):1-137.

16. Virginia Department of Health. STD surveillance network Report. 2013 http://www.vdh.virginia.gov/content/uploads/sites/10/2016/ 01/2013-SSuN-Annual-Report-09-18-13-1.pdf (Accessed 30 Jun 2017).

17. Castro JG, Alcaide ML. High Rates of STIs in HIV-infected patients attending an STI Clinic. South Med J 2016;109:1-4.

18. Berry SA. Gonorrhoea and chlamydia screening in HIV clinics: time for new tools and targets. Sex Transm Infect 2014;90:574-5.

19. Hoover KW, Butler M, Workowski K, et al. STD screening of HIVinfected MSM in HIV clinics. Sex Transm Dis 2010;37:771-6.

20. CDC. PrEP for men who have sex with men. $2018 \mathrm{https} / / /$ prepmaryland.org/sites/default/files/download-resources/PrEP MSM both sides March 2017.pdf.

21. Koenig LJ, Lyles C, Smith DK. Adherence to antiretroviral medications for HIV pre-exposure prophylaxis. Am J Prev Med 2013;44:S91-8.

22. Jenness S, Goodreau S. Impact of the centers for disease control's HIV preexposure prophylaxis guidelines for men who have sex with men in the United States. J Infect Dis 2016;14:1800-7.

23. Vissers DC, Voeten HA, Nagelkerke NJ, et al. The impact of preexposure prophylaxis (PrEP) on HIV epidemics in Africa and India: a simulation study. PLoS One 2008;3:e2077.

24. Bolu OO, Lindsey C, Kamb ML, et al. Is HIV/sexually transmitted disease prevention counseling effective among vulnerable populations?: a subset analysis of data collected for a randomized, controlled trial evaluating counseling efficacy (Project RESPECT). Sex Transm Dis 2004;31:469-74.

25. Carter JW, Hart-Cooper GD, Butler MO, et al. Provider barriers prevent recommended sexually transmitted disease screening of HIV-infected men who have sex with men. Sex Transm Dis 2014:41:137-42.

26. Centers for Disease Control and Prevention. Preexposure prophylaxis for the prevention of hiv infection in the united states-2014: a clinical practice guideline. 2014

27. Calabrese SK, Underhill K. How stigma surrounding the use of hiv preexposure prophylaxis undermines prevention and pleasure: a call to destigmatize "truvada whores". Am J Public Health 2015;105:1960-4.

28. Highleyman L. PrEP use is rising fast in US, but large racial disparities remain. Aidsmap 2016 http://www.aidsmap.com/PrEPuse-is-rising-fast-in-US-but-large-racial-disparities-remain/page/ 3065545/ (Accessed 14 May2018).

29. Clotet B, Feinberg J, van Lunzen J, et al. Once-daily dolutegravir versus darunavir plus ritonavir in antiretroviral-naive adults with HIV-1 infection (FLAMINGO): 48 week results from the randomised openlabel phase 3b study. Lancet 2014;383:2222-31.

30. Kent CK, Chaw JK, Wong W, et al. Prevalence of rectal, urethral, and pharyngeal chlamydia and gonorrhea detected in 2 clinical settings among men who have sex with men: San Francisco, California, 2003. Clin Infect Dis 2005;41:67-74.

31. Lister NA, Smith A, Tabrizi S, et al. Screening for neisseria gonorrhoeae and chlamydia trachomatis in men who have sex with men at male-only saunas. Sex Transm Dis 2003;30:886-9.

32. Sherrard J, Barlow D. Gonorrhoea in men: clinical and diagnostic aspects. Genitourin Med 1996;72:422-6.

33. Morris SR, Klausner JD, Buchbinder SP, et al. Prevalence and incidence of pharyngeal gonorrhea in a longitudinal sample of men who have sex with men: the EXPLORE study. Clin Infect Dis 2006;43:1284-9.

34. Beck EC, Birkett M, Armbruster B, et al. A Data-Driven Simulation of HIV Spread Among Young Men Who Have Sex With Men: Role of Age and Race Mixing and STIs. J Acquir Immune Defic Syndr 2015;70:186-94.
35. Kretzschmar M, van Duynhoven YT, Severijnen AJ. Modeling prevention strategies for gonorrhea and Chlamydia using stochastic network simulations. Am J Epidemiol 1996;144:306-17.

36. Korenromp EL, Sudaryo MK, de Vlas SJ, et al. What proportion of episodes of gonorrhoea and chlamydia becomes symptomatic? Int $J$ STD AIDS 2002;13:91-101.

37. Althaus CL, Heijne JCM, Roellin A, et al. Transmission dynamics of chlamydia trachomatis affect the impact of screening programmes. Epidemics 2010;2:123-31.

38. Price MJ, Ades AE, Angelis DD, et al. Mixture-of-exponentials models to explain heterogeneity in studies of the duration of Chlamydia trachomatis infection. Stat Med 2013;32:1547-60

39. Turner KM, Adams EJ, Gay N, et al. Developing a realistic sexual network model of chlamydia transmission in Britain. Theor Biol Med Model 2006;3:3:3.

40. Chesson HW, Bernstein KT, Gift TL, et al. The cost-effectiveness of screening men who have sex with men for rectal chlamydial and gonococcal infection to prevent HIV Infection. Sex Transm Dis 2013:40:366-71.

41. Tuite AR, Jayaraman GC, Allen VG, et al. Estimation of the burden of disease and costs of genital chlamydia trachomatis infection in Canada. Sex Transm Dis 2012;39:260-7.

42. de Vries R, van Bergen JE, de Jong-van den Berg LT, et al. Systematic screening for chlamydia trachomatis: estimating costeffectiveness using dynamic modeling and dutch data. Value Health 2006:9:1-11.

43. Adams EJ, Turner KM, Edmunds WJ. The cost effectiveness of opportunistic chlamydia screening in England. Sex Transm Infect 2007:83:267-75.

44. Andersen B, Gundgaard J, Kretzschmar M, et al. Prediction of costs, effectiveness, and disease control of a populationbased program using home sampling for diagnosis of urogenital Chlamydia trachomatis Infections. Sex Transm Dis 2006;33:407-15.

45. Gillespie P, O'Neill C, Adams E, et al. The cost and cost-effectiveness of opportunistic screening for chlamydia trachomatis in Ireland. Sex Transm Infect 2012;88:222-8.

46. Roberts TE, Robinson S, Barton PM, et al. Cost effectiveness of home based population screening for Chlamydia trachomatis in the UK: economic evaluation of chlamydia screening studies (ClaSS) project. BMJ 2007;335:291.

47. Welte R, Kretzschmar M, Leidl R, et al. Cost-effectiveness of screening programs for Chlamydia trachomatis: a population-based dynamic approach. Sex Transm Dis 2000;27:518-29.

48. Cohen MS. Sexually transmitted diseases enhance HIV transmission: no longer a hypothesis. Lancet 1998;351:S5-7.

49. Winter AJ, Taylor S, Workman J, et al. Asymptomatic urethritis and detection of HIV-1 RNA in seminal plasma. Sex Transm Infect 1999;75:261-3.

50. Cohen MS, Hoffman IF, Royce RA, et al. Reduction of concentration of HIV-1 in semen after treatment of urethritis: implications for prevention of sexual transmission of HIV-1. AIDSCAP Malawi Research Group. Lancet 1997;349:1868-73.

51. Røttingen JA, Cameron DW, Garnett GP. A systematic review of the epidemiologic interactions between classic sexually transmitted diseases and HIV: how much really is known? Sex Transm Dis 2001;28:579-97.

52. Jin F, Prestage GP, Imrie J, et al. Anal sexually transmitted infections and risk of HIV infection in homosexual men. J Acquir Immune Defic Syndr 2010;53:144-9.

53. Little SJ, McLean AR, Spina CA, et al. Viral dynamics of acute HIV-1 infection. J Exp Med 1999;190:841-50.

54. Leynaert B, Downs AM, de Vincenzi I. Heterosexual transmission of human immunodeficiency virus: variability of infectivity throughout the course of infection. European Study Group on Heterosexual Transmission of HIV. Am J Epidemiol 1998;148:88-96.

55. Beyrer C, Baral SD, van Griensven F, et al. Global epidemiology of HIV infection in men who have sex with men. Lancet 2012;380:367-77.

56. Alam SJ, Meyer R, Norling E. A model for HIV spread in a South African village. Multi-Agent-Based Simul IX 2009:33-45.

57. Baggaley RF, Ferguson NM, Garnett GP. The epidemiological impact of antiretroviral use predicted by mathematical models: a review. Emerg Themes Epidemiol 2005;2:9.

58. Lundgren JD, Babiker AG, Gordin F, et al. Initiation of antiretroviral therapy in early asymptomatic HIV infection. N Engl J Med 2015;373:795-807.

59. Kitahata MM, Gange SJ, Abraham AG, et al. Effect of early versus deferred antiretroviral therapy for HIV on survival. N Engl $J$ Med 2009:360:1815-26. 
60. Palella FJ, Delaney KM, Moorman AC, et al. Declining morbidity and mortality among patients with advanced human immunodeficiency virus infection. N Engl J Med Overseas Ed 1998;338:853-60.

61. Wit FW, Blanckenberg DH, Brinkman K, et al. Safety of long-term interruption of successful antiretroviral therapy: the ATHENA cohort study. AIDS 2005;19:345-8.

62. Maggiolo F, Ripamonti D, Gregis G, et al. Effect of prolonged discontinuation of successful antiretroviral therapy on CD4 T cells: a controlled, prospective trial. AIDS 2004;18:439-46.

63. Ortiz GM, Wellons M, Brancato J, et al. Structured antiretroviral treatment interruptions in chronically HIV-1-infected subjects. Proc Natl Acad Sci U S A 2001;98:13288-93.

64. El-Sadr WM, Lundgren J, Neaton JD, et al. CD4+ countguided interruption of antiretroviral treatment. $N$ Engl $J$ Med 2006;355:2283-96.

65. Hill T, Bansi L, Sabin C, et al. Data linkage reduces loss to follow-up in an observational HIV cohort study. J Clin Epidemiol 2010;63:1101-9.
66. Mocroft A, Kirk O, Aldins P, et al. Loss to follow-up in an international, multicentre observational study. HIV Med 2008;9:261-9.

67. Ndiaye B, Ould-Kaci K, Salleron J, et al. Characteristics of and outcomes in HIV-infected patients who return to care after loss to follow-up. AIDS 2009;23:1786-9.

68. Mills EJ, Nachega JB, Buchan I, et al. Adherence to antiretroviral therapy in sub-Saharan Africa and North America: a meta-analysis. JAMA 2006;296:679-90.

69. Rana Al, Liu T, Gillani FS, et al. Multiple gaps in care common among newly diagnosed HIV patients. AIDS Care 2015;27:679-87.

70. Rosenberg ES, Millett GA, Sullivan PS, et al. Understanding the HIV disparities between black and white men who have sex with men in the USA using the HIV care continuum: a modeling study. Lancet HIV 2014;1:e112-8.

71. Maryland Department of Health and Mental Hygiene, MDHMH. 2012 Baltimore city annual hiv epidemiological profile. Baltimore: $\mathrm{MDHMH}$ 2012. 